\title{
Encapsulated Objects for Neural Network Simulation
}

\author{
Mark A. Rubin ${ }^{1}$ \\ Physics Division, Naval Air Warfare Center ${ }^{2}$ \\ China Lake, California 93555 \\ Physics Department, Rockefeller University \\ New York, New York 10021
}

\begin{abstract}
We consider aspects of program design relevant to the creation of object-oriented software tools for research in neural network algorithms, and discuss these in the context of EONNS, a set of $\mathrm{C}++$ classes for neural networks which is currently under development.
\end{abstract}

\footnotetext{
${ }^{1}$ email: mark@peewee.chinalake.navy.mil

${ }^{2}$ mailing address
} 